Revista científica, arbitrada e indizada, bajo la modalidad electrónica.

Recibido: 23/01/2020

Aceptado: 04/02/2020

\title{
ESTRATEGIA, PROSPECTIVA Y SOSTENIBILIDAD COMO ENFOQUES GERENCIALES PARA ALCANZAR LA PRODUCTIVIDAD Y COMPETITIVIDAD DE LAS MIPYMES EN UN MERCADO GLOBAL
}

Strategy, foresight and sustainability as managerial approaches that achieve the productivity and competitiveness of Smesms in a global market

\author{
Juan Carlos Orejuela Vega \\ Universidad Tecnológica del Chocó Diego Luis Córdoba \\ jcov5000@hotmail.com \\ Harlem Sirlein Beltrán Moreno \\ Universidad Tecnológica del Chocó Diego Luis Córdoba \\ harsibemo@yahoo.es \\ Colombia
}

\section{RESUMEN}

En la actualidad, las micro, pequeñas y medianas empresas se encuentran ante un entorno empresarial globalizado regido constantemente por los cambios económicos, sociales, políticos, culturales, tecnológicos y ambientales. De allí, que el reto para los empresarios o gerentes consiste en incorporar enfoques gerenciales, como las estrategias, la prospectiva y la sostenibilidad para alcanzar la productividad y competitividad de las Mipymes en un mercado global. Frente a este propósito, las estrategias actúan como patrones para la toma de decisiones que determinan los objetivos, generan planes y políticas para el logro de estos, considerando para este fin los factores económicos, al igual humanos en beneficio de los grupos de interés. Así mismo, se deben concentrar en la prospectiva, ya que esta permite que la empresa pueda anticiparse al futuro, generar métodos y técnicas para la construcción de escenarios, que permiten minimizar el fenómeno de incertidumbre por medio de la planeación estratégica. Igualmente, se tuvo en cuenta el concepto de sostenibilidad como otro enfoque gerencial, el cual busca la inclusión social, optimizar los recursos naturales y reducir el impacto sobre el medio ambiente, preservando la integridad del planeta para las generaciones futuras, sin descuidar la viabilidad económica y financiera de las empresas.

Palabras clave: Estrategia; prospectiva; sostenibilidad; productividad; competitividad; Mipymes. 


\title{
Revista científica, arbitrada e indizada, bajo la modalidad electrónica.
}

\begin{abstract}
Currently, micro, small and medium-sized companies are facing a globalized business environment constantly governed by economic, social, political, cultural, technological and environmental changes. Hence, the challenge for entrepreneurs or managers is to incorporate management approaches, such as strategies, foresight and sustainability to achieve MSMEs' productivity and competitiveness in a global market. Faced with this purpose, strategies act as patterns for decision-making that determine objectives, and generate plans and policies to achieve them, considering for this purpose economic and human factors for the benefit of interest groups. Likewise, they should focus on foresight, since this allows the company to anticipate the future, generate methods and techniques for the construction of scenarios, which allow minimizing the phenomenon of uncertainty through strategic planning. Likewise, the concept of sustainability was taken into account as another management approach, which seeks social inclusion, optimizing natural resources and reducing the impact on the environment, preserving the integrity of the planet for future generations, without neglecting economic viability. And financial of the companies.
\end{abstract}

Keywords: strategies, foresight, sustainability, productivity, competitiveness, Mipymes.

\section{INTRODUCCIÓN}

El desarrollo de las micro, pequeñas y medianas empresas (Mipymes) dentro la globalización de los mercados, guiada de las respuestas efectivas de aquellas empresas buscando su crecimiento dentro de escenarios internacionales, además las tendencias como las nuevas tecnologías de la información, hacen que continuamente aparezcan nuevos escenarios marcando la oferta y la demanda referida a la incorporación constante de nuevos enfoques gerenciales, los cuales permiten el aprovechamiento de las vías de negocios que sugieren las condiciones de los mercados emergentes.

De esta manera, las Mipymes se encuentran ante un entorno empresarial globalizado regido constantemente por los cambios económicos, sociales, políticos, culturales tecnológicos y ambientales. Estos, por consiguiente, han llevado a que las empresas que tienen como objetivo fundamental el crecimiento, orienten sus miradas hacia los mercados extranjeros, pues la globalización de la mano con sus fenómenos económicos además sociales, presenta una amplia gama de posibilidades, igual proporciona el contexto para alcanzar este propósito. 


\section{Revista científica, arbitrada e indizada, bajo la modalidad electrónica.}

Por consiguiente, el crecimiento empresarial se asocia directamente con el resultado en interrelación de las fuerzas externas, además las capacidades desarrolladas por las Mipymes, en este sentido, las condiciones actuales del mercado, al igual la búsqueda constante de nuevos ingresos, llevan a las pequeñas y medianas empresas a intensificar la exploración de nuevas alternativas que les permitan crecer, mejorando así los efectos de la competencia global. De esta manera, se vuelve importante adoptar enfoques gerenciales como medio de crecimiento, que permitan mejorar la visión global, la productividad e innovación; es decir, garantizar una condición de éxito para entrar a los mercados extranjeros y mantener una condición de permanencia.

De allí, el propósito de este estudio es de una manera exploratoria y descriptiva analizar las estrategias, la prospectiva, además de la sostenibilidad como enfoques gerenciales para alcanzar la productividad, al igual competitividad de las Mipymes en un mercado global, puesto el desarrollo de estos dos factores, representados en rendimiento y capacidad, se convierten en el principal reto de las pequeñas y medianas empresas. Esto se debe a que los mercados internacionales son cada vez más complejos debido a fenómenos como la competencia, la elevada revolución económica, los constantes cambios tecnológicos, el alto nivel de incertidumbre, las transformaciones, la generalización de conocimientos, la cobertura comunicacional y la integración.

Con respecto a lo planteado, las micro, pequeñas, así como las medianas empresas son cada vez más conscientes de la necesidad de desplegar capacidades, al igual competencias que hagan posible aumentar geográficamente su actividad comercial, generando productos y servicios para satisfacer de manera rentable insuficiencias dentro del mercado consumidor, para crecer y hacerse sostenibles en el tiempo. En consecuencia, es ante este tipo de situaciones en las cuales la incorporación de estrategias, prospectiva y sostenibilidad se convierten en herramientas gerenciales idóneas para generar y fortalecer los diferentes recursos y procesos que deberán incorporarse en las Mipymes para tomar decisiones que conduzcan a alcanzar la productividad y competitividad en los diferentes procesos que exige la participación y permanencia en un mercado global. 


\section{Revista científica, arbitrada e indizada, bajo la modalidad electrónica.}

\section{MARCO TEÓRICO}

\section{Contexto de las pequeñas y medianas empresas dentro del mercado global}

Dentro del marco de análisis para este estudio, una investigación de las Naciones Unidas y la Comisión Económica para América Latina y el Caribe (CEPAL) sobre los nuevos desafíos de fomento para América Latina, así como los países del Caribe, llevada a cabo en 2018, permitió conocer que el sector privado comprendido principalmente por las micro, pequeñas y medianas empresas (Mipymes) es el mayor ente empleador en el sector productivo de estas naciones. Fue posible evidenciar que su peso en el tejido productivo representa el 99\% del total del parque empresarial formal existente en dichos países.

Por consiguiente, las Mipymes concentran el 61\% del empleo privado en estos países, al igual una participación al producto interno bruto (PIB) de tan solo el 25\%, lo cual las convierte en un actor central para garantizar la viabilidad, al igual eficacia de la transformación generadora de una nueva dinámica de desarrollo, que permita un crecimiento económico más rápido y continuo para la economía de estos países, pero que al mismo tiempo sea incluyente y sostenible.

Ahora bien, en atención a las consideraciones referidas en este estudio, los datos son bajos comparados con países como Italia donde las Mipymes participan con el 52\% del crecimiento económico del PIB y Corea del Sur (55\%). Por su parte, en Francia, Holanda, Finlandia, al igual en Suecia es mayor al 47\%. En cuanto a la generación de empleo, se puede afirmar que en Estados Unidos las Mipymes representan el 76\% del empleo; en Alemania, Bélgica, Canadá, Reino Unido y Francia, está cerca al 66\%; en países como Dinamarca, Portugal, España, Grecia, entre otros, es del 80\%. Cabe aclarar que los datos son tomados antes de la crisis socioeconómica generada por la pandemia del Covid - 19.

En este sentido, se logró conocer cómo los emprendedores o gerentes de estos entes de la producción consideran saberlo todo, desconocen el entorno, suponen que todo está inventado y toman pocas decisiones basadas en procesos de planificación. Para ellos, todo producto se vende solo, creen en la base de ser pionero como la forma para alcanzar el éxito. Igualmente, no tiene el conocimiento para comenzar la actividad empresarial, 


\section{Revista científica, arbitrada e indizada, bajo la modalidad electrónica.}

comercializan sin establecer un plan mercadotécnico, tienen resistencia al cambio, presentan excesivos riesgos, carecen de análisis sobre la viabilidad y factibilidad en la ejecución estratégica del marketing.

Así mismo, estos informes permiten evidenciar cómo los emprendedores no planifican lo suficiente sobre acciones concretas, carecen de liderazgo en la toma de decisiones, presentan una aplicación limitada en los métodos prospectivos orientados a ser más proactivos que reactivos y establecen procesos limitados para el seguimiento de las actividades realizadas por estos emprendedores. Ahora bien, se puede notar cómo la fijación de los precios se realiza sin la estructuración en los costos, al considerar poco fundamental la planificación financiera e interpretación de estos, ya sea en producción, en ventas, utilidades, márgenes, entre otros.

Por lo tanto, estos aspectos simbolizan frecuentes debilidades en torno a los emprendedores de las Pymes. No obstante, como causas que generan esta situación, fueron detectados algunos fenómenos relacionados con la globalización, internacionalización económica, los tratados de libre comercio, la situación del país en relación con la poca seguridad jurídica, la inflación, las políticas fiscales, la corrupción, la conflictividad social e inseguridad, los acelerados cambios normativos, el bajo desarrollo económico e industrial, y la deficiencia en servicios públicos y obras en infraestructura vial. En consecuencia, es evidente el descontrol en la entrada de nuevos productos, así como competidores extranjeros.

También se deben tener en cuenta las operaciones y costumbres tradicionalistas realizadas por las Pymes al estar constituidas o gerenciadas por familiares. Esto puede ocasionar que no consideren como importante la innovación en los avances tecnológicos, las capacitaciones en el manejo administrativo, tanto técnico como operativo; planificar sobre la realidad, dejando atrás creencias culturales o ancestrales, y solo vender lo que realmente necesita el cliente, mas no lo ofertado por estas entidades. Del mismo modo, es conveniente considerar que, sin empresas competitivas, es poca la posibilidad de alcanzar el crecimiento y desarrollo económico; sin crecimiento económico, es difícil superar la pobreza; y sin empleo productivo, hay limitada posibilidad para el crecimiento. Por lo anterior, la dinamización a partir del talento de los emprendedores 


\section{Revista científica, arbitrada e indizada, bajo la modalidad electrónica.}

dentro las Pymes podría ser una estrategia idónea para alcanzar prosperidad socioeconómica para las economías domésticas vulnerables.

Aquí se entra a entender cómo el fenómeno de la globalización ha generado grandes tendencias, como la innovación, la forma de producir bienes y servicios, los acelerados cambios tecnológicos, la revolución de las telecomunicaciones, la creciente interdependencia económica, los cambios en los gustos y preferencias de los consumidores, entre otros factores que se convierten en fuentes claves para generar tanto oportunidades como amenazas en la búsqueda de productividad y competitividad de las pequeñas y medianas empresas. Por consiguiente, la adopción e implementación de estrategias y el uso de la prospectiva y la sostenibilidad (económica, social, ambiental y gerencial) entran a hacer parte de herramientas idóneas para su posicionamiento, crecimiento y desarrollo económico, ya que permiten la adaptación a las exigencias presentes en el mercado nacional como internacional.

\section{Las estrategias, la prospectiva y la sostenibilidad como enfoques gerenciales} para alcanzar la productividad y competitividad de las Mipymes en un mercado global.

Concepción de las estrategias como herramientas gerenciales para el mejoramiento continúo.

Es de entender que dentro de un mercado altamente globalizado la búsqueda de la productividad y la competitividad por parte de las Mipymes exige la apropiación de enfoques gerenciales, que les permitan servir como herramientas idóneas para mantener su participación y crecimiento en relación con la producción de bienes y servicios, que de forma rentable satisfagan necesidades y deseos en los consumidores o usuarios, diferenciándose así de su competencia. Para expertos como Arango (2005), la capacidad de las empresas para obtener resultados superiores depende fundamentalmente de las habilidades gerenciales para adquirir y coordinar recursos, y percibir la información para la toma de decisiones.

Para Tarapuez, Guzmán y Hernández (2016), las estrategias se han convertido en la base para promover el crecimiento, la productividad y la competitividad. Son la 


\section{Revista científica, arbitrada e indizada, bajo la modalidad electrónica.}

herramienta fundamental para el éxito y la sostenibilidad de las empresas que han logrado perdurar; sin embargo, desde los años sesenta comenzaron a aparecer nuevos términos, como gestión y administración estratégica, y es ahí cuando la estrategia se empieza a convertir en un proceso lógico y consciente, que contribuye a la labor de la gerencia.

Por su parte, Andrews, citado por Tarapuez et al., (2016), afirma que las estrategias son patrones para la toma de decisiones que determinan los objetivos y metas, lo que genera planes y políticas para el logro de los mismos, teniendo en cuenta para este fin los factores económicos y humanos en beneficio de los accionistas, empleados, clientes y comunidad. No obstante, para Beaver (2007), las estrategias orientan las decisiones, permitiendo que los planes operativos se realicen de forma concurrente al logro de los objetivos de la empresa, con la finalidad de incrementar las posibilidades del cumplimiento de las metas. Al respecto, Burgelman (2002), define las estrategias como la teoría que la alta dirección tiene sobre la base de sus éxitos pasados y futuros.

Por su parte, Miles y Snow (2003), afirman que de las estrategias depende el principal direccionamiento para que las empresas sean clasificadas como inactivas, reactivas, preactivas y proactivas. De esta manera, los procesos estratégicos implican necesariamente que las empresas tengan una planificación a largo plazo, apoyada en procesos formales en los cuales se definan metas, objetivos, programas, actividades, recursos, entre otros, y que además consideren la dinámica del ambiente en el cual operan y los continuos cambios que se presentan en el entorno. Adicionalmente, la articulación adecuada de un proceso formal de formulación, implementación y control estratégico, y la adaptación a los continuos cambios exige que las empresas identifiquen sus capacidades esenciales o competencias centrales.

Así, desde la perspectiva económica y del entorno de los negocios, las estrategias están más relacionadas con la capacidad de las empresas para responder a los cambios y exigencias del mercado. Para Hofery (citado por Tarapuez et al., (2016), la estrategia es una de las principales herramientas de la alta gerencia para hacer frente a los cambios, tanto externos como internos. De esta manera, hará parte de cómo una empresa aprovecha sus 


\section{Revista científica, arbitrada e indizada, bajo la modalidad electrónica.}

fortalezas para distinguirse de forma positiva de sus competidores, dependiendo igualmente del cliente y el entorno.

Por consiguiente, las estrategias como enfoque gerencial se convierten en guía o plan de tareas, que ordenadas por las metas, políticas y valores de las empresas permiten el mejoramiento continuo dentro de la materialización de los objetivos y la misión de la compañía. De esta manera, actúa como generadora de ventajas competitivas, lo que a su vez facilita la obtención y el direccionamiento de recursos, y fortalecimientos dentro de ambientes altamente competitivos. Por lo anterior, la estrategia es un concepto utilizado en muchos contextos, y en las Mipymes se ha convertido en la base para promover el crecimiento, la productividad y la competitividad.

\section{Las estrategias como herramienta para alcanzar la productividad y la} competitividad de las Mipymes dentro del mercado global

Desde esta perspectiva, la formulación, la implementación y el control de la estrategia exige a las empresas tomar decisiones asertivas para su crecimiento y mejora continua, por lo cual Jones y George (2006), conciben la estrategia como un conglomerado de decisiones relativas a qué metas perseguir, qué actividades emprender y cómo aprovechar los recursos para alcanzarlas. De allí que estudios relacionados con las estrategias como herramientas de mejoramiento continuo para las organizaciones o empresas han considerado que priorizar de manera táctica la estructura organizacional, la innovación y el liderazgo juega un papel determinante para el alcance de la productividad y competitividad empresarial.

Para Ponti (2009):

Las estructuras en las organizaciones, determinan la forma en que estas establecen sus procesos y operaciones cotidianas; aunque existen diferentes tipos de estructuras, en general estas deben estar orientadas a apoyar la estrategia. Ya que las empresas primero deben plantear una estrategia y a partir de ella poder diseñar una estructura. Si una empresa está demasiado jerarquizada, probablemente tendrá importantes dificultades para innovar o generar ideas que coadyuven al logro de la productividad y competitividad empresarial. (p. 96) 


\section{Revista científica, arbitrada e indizada, bajo la modalidad electrónica.}

Por el contrario, en una estructura donde prevalece la autoridad, afectará la comunicación y las relaciones de confianza para dar soluciones creativas a los nuevos retos y para generar cambios. Al respecto, Wheelen y Hunger (2013), señalan "que una estructura eficaz en una organización, soporta la estrategia; por consiguiente, la estructura se debe modificar si solo es para volverla más avanzada y acorde con los desafíos propuestos en la estrategia organizacional” (p. 162).

Otro factor de vital importancia en este estudio es el liderazgo, concepto que ha sido ampliamente estudiado por diversos autores a lo largo de la historia. Su aplicación exige una serie de características personales y del entorno; sin embargo, por el objetivo a alcanzar dentro de este estudio, solo se abordará desde la perspectiva de ser un aspecto importante dentro del alcance del mejoramiento continuo para las micro, pequeñas y medianas empresas, haciendo énfasis en la importancia de estimular el liderazgo participativo en los empleados como condición fundamental para fomentar la creatividad y la innovación mediante el trabajo en equipo que requieren las Mipymes para alcanzar su productividad y competitividad en mercados globales.

Para Vroom (2003), el carácter de empresas innovadoras y con alta capacidad de adaptación al cambio debe contar con políticas orientadas a estimular la participación de las personas en los distintos procesos y operaciones cotidianas. Por consiguiente, el estilo de liderazgo participativo invita a los empleados a que aporten en los procesos de toma de decisiones organizacionales. El liderazgo y la participación de los empleados adopta como premisa el diseño y la construcción de los diferentes tipos de estructuras y actividades cotidianas de las empresas.

Una investigación sobre los estilos de liderazgo realizada por White y Lippit (citados por Chiavenato, 2009), permitió conocer las ventajas del liderazgo democrático; es decir, ser extremadamente comunicativo, alentar a la participación de las personas por ser justo y no arbitrario, y por ocuparse tanto del problema de las personas como del trabajo mismo.

En relación con la innovación, esta actúa como un proceso ordenado y coherente con la planificación estratégica de la empresa, en el que las estrategias y las políticas juegan un papel fundamental a la hora de ejecutar las acciones que conllevan la productividad y competitividad de las empresas. 


\section{Revista científica, arbitrada e indizada, bajo la modalidad electrónica.}

Para Drucker citado por Álvarez (2014), la innovación no es solo producto de los cambios tecnológicos ni del resultado de la investigación y del desarrollo, sino que las innovaciones más importantes se deben hacer de una forma social y organizacional, ya que es el cambio lo que proporciona la oportunidad a lo nuevo y lo diferente. Debido a esto, lo primero que se hace es identificar los diferentes cambios que se han producido en las empresas y dejar atrás lo que fue creado ayer, en vez de seguir defendiéndolo, para así darle una oportunidad a la generación de nuevas ideas que conduzcan al desarrollo continuo de nuevos productos, servicios y procesos.

En consecuencia, sostiene Oslo (2012), que la innovación no es solo la concepción e implantación de cambios producidos al producto, sino además de cambios que se le deben realizar al marketing, al proceso y a la organización de la empresa, teniendo como finalidad el mejoramiento de los resultados. Estos cambios innovadores pueden ser realizados mediante la adecuación de tecnología desarrollada adquirida, interna o externamente, y la aplicación de nuevos conocimientos que permitan crear nuevos proyectos para el mercado. No obstante, para que estas actividades de innovación se lleven a cabo deben incluirse las actuaciones científicas, organizativas, tecnológicas, comerciales y financieras, para así hacer un estudio de la viabilidad y el éxito de la innovación.

De esta manera, el propósito de la estrategia es desarrollar una ventaja competitiva sostenible que dure el mayor tiempo posible asociado con la posesión de competencias fundamentales, y que a partir de sus habilidades organizacionales las empresas puedan aplicarlas a productos, clientes o mercados, y convertirlas, por lo tanto, en rentabilidad para sus propietarios.

Para Landázuri y Montenegro (2018), el desarrollo y el crecimiento de las empresas depende de las estrategias que se formulen e implementen para generar ventaja competitiva. Culkin \& Smith (2000) manifiestan que "a diferencia de una gran empresa, el administrador de una Mipymes no puede tomar decisiones complejas por medio de canales especializados, sino que es el administrador o emprendedor quien asume la responsabilidad de tomar decisiones sobre tecnología, comunicaciones, banca, papelería, entre otros” (p. 7).

De igual manera, Culkin \& Smith (2000), expresan que para las empresas, el negocio está ligado a la vida e identidad del empresario, en las cuales, en razón a la condición 


\section{Revista científica, arbitrada e indizada, bajo la modalidad electrónica.}

presente en la gestión de las Mipymes, es importante resaltar cuál o cuáles estrategias son aplicadas por los empresarios en este caso, el sector de consumo masivo, con el fin de generar ventajas competitivas, alcanzar el éxito y lograr la permanencia en el mercado.

Porter (citado por Landázuri y Montenegro, 2017), sostiene que la ventaja competitiva radica en las muchas actividades discretas que desempeña una empresa con respecto al diseño, la producción, el marketing, las finanzas, entre otras. Cada una de estas actividades puede contribuir a la posición de costos relativos de las empresas y crear una base para la diferenciación. Es así que diversos autores acogen la teoría de las ventajas competitivas, relacionando el crecimiento de las empresas con las estrategias genéricas planteadas por Porter, que son: liderazgo en costos, diferenciación y enfoque o concentración.

\section{Concepción de la prospectiva como herramienta gerencial para el mejoramiento continúo}

Para Godet (2011), la prospectiva llamada foresight proveniente de la palabra prospectus, que significa "mirar hacia adelante" está catalogada como una ciencia utilizada como herramienta para los estudios de anticipación del futuro. Mediante la prospectiva también se pueden encontrar métodos y técnicas para la construcción de escenarios y pronósticos, la creación de modelos y la contribución para la toma de decisiones en el presente. Todo esto tiene un alcance enorme, que abarca la totalidad de los distintos aspectos de la vida del ser humano desde lo político, económico, social o personal.

Medina \& Ortegón (2006), sostienen que la prospectiva es aplicable a todos los ámbitos de la vida cotidiana, es una herramienta esencial para sectores como el tecnológico, medioambiental, económico, territorial, político, humano, social, arquitectónico, empresarial y otros más. En el campo organizacional o empresarial, es una herramienta para la toma de decisiones, tanto en el sector público como en las empresas privadas, desde el punto de vista de la ciencia, tecnología e innovación. Específicamente dentro del sector empresarial, actúa como un instrumento que facilita la mejora continua en los procesos, lo que permite que las Mipymes minimicen el fenómeno de incertidumbre y orienten sus 


\section{Revista científica, arbitrada e indizada, bajo la modalidad electrónica.}

acciones a través de planeación estratégica a largo plazo, con el propósito de alcanzar objetivos planteados previamente.

Es importante entender que, en relación con el propósito de este estudio, la prospectiva no tiene la intensión de pronosticar el futuro de las micro, pequeñas y medianas empresas, su papel está relacionado con la meditación sobre los acontecimientos que podrían suceder dentro de su porvenir. Por consiguiente, entra a convertirse en una herramienta de previsión, en la cual, por medio de planificación estratégica, los gerentes o emprendedores podrán generar las acciones pertinentes a favorecer la adopción de recursos $\mathrm{y}$ procesos que hagan posible su productividad y competitividad dentro del mercado nacional y global.

\section{La prospectiva como herramienta para alcanzar la productividad $y$ competitividad de las Mipymes dentro del mercado global}

Para conocer la prospectiva como herramienta para alcanzar la productividad y competitividad de las Mipymes, es importante partir del aporte de Mojica (2010), en el cual sustenta que los empresarios deben analizar variables relacionadas con el porvenir de las empresas, con el fin de escoger las estrategias o acciones más apropiadas para alcanzar el objetivo establecido. Es así que al realizar el estudio indicado, se escoge la mejor opción y, además, se formulan estrategias para alcanzar las metas y obtener un mayor porcentaje de éxito. Por medio de la prospectiva empresarial u organizacional, se puede alcanzar una ventaja competitiva frente a otras empresas.

Medina, Becerra y Castaño (2014), afirman que la prospectiva empresarial u organizacional establece un método para el buen manejo de la toma de decisiones. Este método incluye el estudio de un entorno general, la investigación de nuevos mercados, la actualización y el desarrollo de nuevos productos y servicios. Por consiguiente, la aplicación de esta prospectiva supone el trabajar con las capacidades humanas de exploración, descubrimiento y experimentación. Con este método se puede corregir y evitar riesgos u obstáculos que se puedan presentar en el futuro, frente a la generación y fortalecimiento de sus ventajas competitivas. 


\section{Revista científica, arbitrada e indizada, bajo la modalidad electrónica.}

Por su parte, Popper (2008), establece que la metodología es un grupo de procedimientos que siguen un orden establecido dentro de un estudio científico. Los métodos hacen uso de varias herramientas para alcanzar metas establecidas por la empresa u organización. En el ámbito de la prospectiva, existe una infinidad de métodos y herramientas, por lo cual, para comprender mejor las metodologías de la prospectiva utilizadas como herramientas para alcanzar la productividad y competitividad empresarial, Medina et al., (2014) propone el siguiente diamante de métodos (Figura 1).

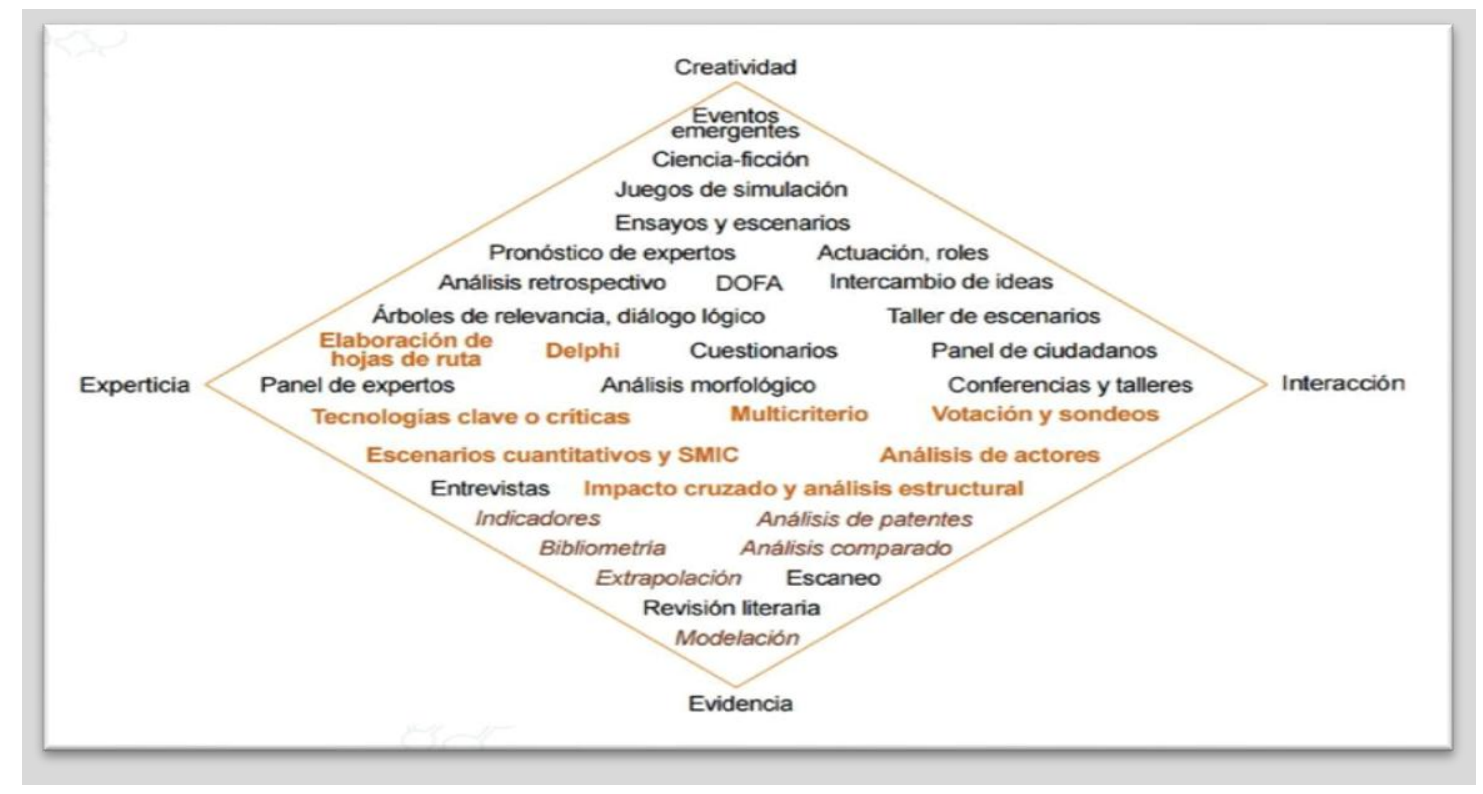

Figura 1. Diamante de métodos prospectivos para la toma de decisiones. Fuente: (Medina et al., 2014, p.81)

Igualmente, dentro de la prospectiva se encuentran otros métodos que son cualitativos y cuantitativos, cada uno de ellos tiene sus respectivas herramientas. Para Medina y Ortegón (2006), las herramientas de prospectiva pueden ser usadas en diferentes ámbitos dependiendo del nivel de incertidumbre que tengan. Así, para Baena (2004) las herramientas clasificadas como cualitativas se desarrollan a partir de opiniones y juicios de profesionales en los temas de estudio. Se podría decir que este es un método subjetivo y personal. 


\section{Revista científica, arbitrada e indizada, bajo la modalidad electrónica.}

Así mismo, para Popper (2008), las herramientas cuantitativas son aquellas que ayudan a fabricar tendencias partiendo de datos reales, estas herramientas toman en cuenta el pasado y lo integran para crear un criterio completo de una situación y de esa manera poder prever el futuro. Para la aplicación de estos métodos, se utiliza de manera frecuente la tecnología y la informática (Tabla 1).

Tabla 1. Herramientas prospectiva cualitativas y cuantitativas para la toma de decisiones.

\begin{tabular}{|c|c|}
\hline $\begin{array}{l}\text { Herramientas prospectivas } \\
\text { cualitativas }\end{array}$ & $\begin{array}{l}\text { Herramientas prospectivas } \\
\text { cuantitativas }\end{array}$ \\
\hline $\begin{array}{ll}\text { - } & \text { Juego de actores } \\
\text { - } & \text { Análisis morfológico } \\
\text { - } & \text { SmicProbExpert } \\
\text { - } & \text { Ábaco de Régnier } \\
\text { - } & \text { Ejes de Schwartz } \\
\text { - } & \text { Escalas de futuro }\end{array}$ & 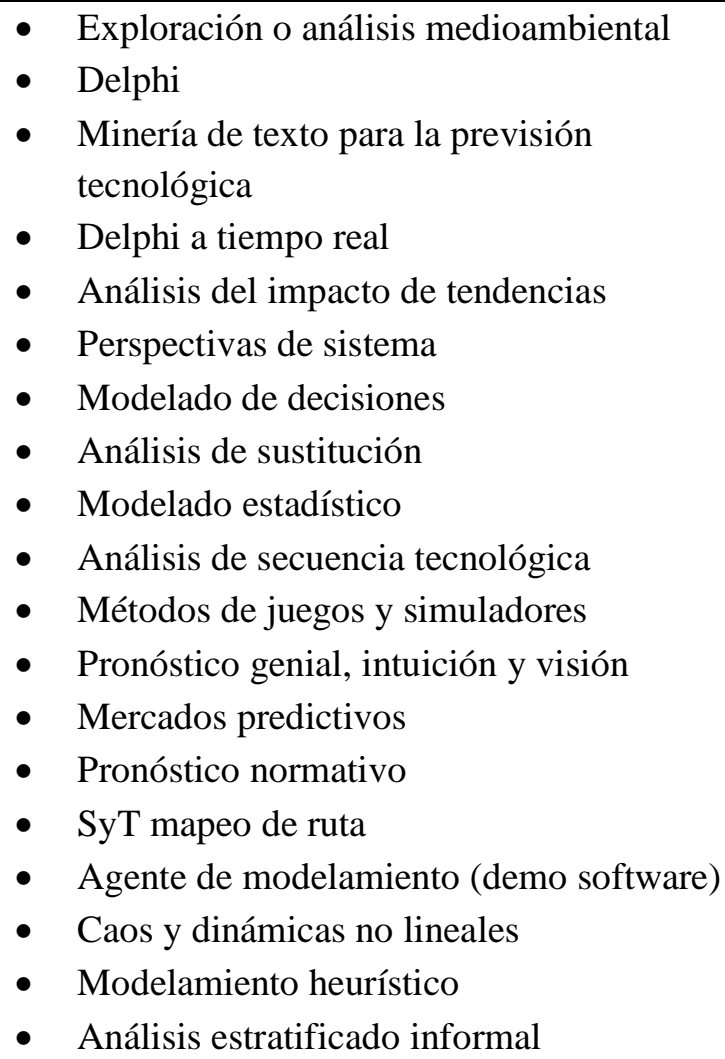 \\
\hline Fuente: Baena (2004) & Fuente: Popper (2008) \\
\hline
\end{tabular}

Concepción de la sostenibilidad como herramienta gerencial para el mejoramiento continuo 


\section{Revista científica, arbitrada e indizada, bajo la modalidad electrónica.}

Es importante determinar que cuando se incorpora el concepto de sostenibilidad dentro del proceso productivo o empresarial, no se debe asociar con el de responsabilidad social corporativa, ni mucho menos limitarla a la obtención de un equilibrio aceptable entre balance económico, social y ambiental. La sostenibilidad como herramienta gerencial para el mejoramiento continuo se relaciona, de acuerdo con Sánchez (2012), con una oportunidad de cambio y transición hacia una nueva forma de hacer negocios, pero también hacia nuevas formas de convivir con una sociedad y el medio que la rodea.

De esta manera, para el sector empresarial, el concepto de sostenibilidad representa un nuevo enfoque para hacer negocios. Al desarrollar sus actividades, las empresas promueven la inclusión social, optimizan la utilización de los recursos naturales y reducen el impacto sobre el medio ambiente, preservando así la integridad del planeta para las generaciones futuras, sin despreciar su viabilidad económica y financiera.

Para Pitre et al., (2017), el emprendimiento sostenible para el desarrollo económico es visto como un conjunto de acciones creativas que se direccionan en la búsqueda de mejorar una situación crítica o de construir algo nuevo independientemente de los recursos disponibles en el medio. Las acciones creativas que llevan a emprender y que dependen exclusivamente del capital humano son las encargadas de proyectar ideas estratégicas capaces de transformar situaciones que alteran el buen funcionamiento de las iniciativas de negocios.

Por consiguiente, Chirinos et al., (2017), manifiestan que la sostenibilidad en las empresas es una forma de organización que permite el avance económico y el desarrollo local, trayendo consigo generación de empleo y la creación de bienes y servicios que aportan al crecimiento de las comunidades donde se desarrollen las iniciativas de negocios. En consecuencia, proporciona rentabilidad para el emprendedor e impulsa el mercado de competencias donde se identifique la oferta y la demanda, es decir que los consumidores tengan posibilidad de elegir entre un competidor y otro, para estabilizar la economía dentro del mercado.

La concepción de la sostenibilidad como herramienta gerencial para el mejoramiento continuo actúa como un sistema que conduce al mejor uso de los recursos ambiéntales, la 


\section{Revista científica, arbitrada e indizada, bajo la modalidad electrónica.}

gestión del talento humano, la innovación, la cohesión social y la mejora en los productos y servicios dentro del mercado, lo que proporciona beneficios, tanto para la empresa como para la sociedad.

Según los aportes de Dean \& McMullen (2007), el emprendimiento sostenible es el proceso de descubrir, evaluar y aprovechar las oportunidades económicas presentes en las fallas del mercado cuando hay una inapropiada asignación de recursos que obstaculizan la sostenibilidad, incluyendo las que son relevantes para el medio ambiente, como bienes públicos, externalidades, el poder de monopolio, la inapropiada intervención del gobierno e información imperfecta Así, para el desarrollo del emprendimiento sostenible es necesario superar las barreras del funcionamiento eficiente de los mercados.

\section{La sostenibilidad como herramienta para alcanzar la productividad y} competitividad de las Mipymes dentro del mercado global

La sostenibilidad es un concepto muy amplio, complejo y multidimensional para la cual no hay una receta única ni se puede resolver con una sola acción. Ante esta nueva realidad, es necesario plantear nuevos modelos de gestión, no solo de las empresas, sino de sus objetivos y estrategias para que la prosperidad de estas sea compatible con una calidad de vida sostenible a nivel planetario. Chirinos et al., (2017), sostienen que propósitos como el de consolidar la sostenibilidad como herramienta para alcanzar la productividad y competitividad de las Mipymes dentro del mercado global, entra hacer de los nuevos paradigmas que algunos autores llaman capitalismo natural y se basa en cuatro principios; aumentar la eficacia de los recursos, eliminar el concepto de desecho, modificar la estructura económica de una economía a base de industrias a una economía de servicios y por último invertir en regeneración natural.

Sin embargo, estos siguen siendo aspectos muy amplios y difíciles de concretar en las empresas. Por consiguiente, hay muchos factores que influyen a la hora de obtener sostenibilidad empresarial, y estos se pueden dividir en factores externos los que están fuera del estricto control empresarial e internos (Tabla 2).

Tabla 2. Factores que influyen a la hora de obtener sostenibilidad empresarial 


\section{Revista científica, arbitrada e indizada, bajo la modalidad electrónica.}

\begin{tabular}{|l|l|}
\hline \multicolumn{1}{|c|}{ Factores externos } & \multicolumn{1}{|c|}{ Factores internos } \\
\hline Precios de materias primas & Políticas ambientales de la empresa \\
\hline Servicios públicos & $\begin{array}{l}\text { Eficiencia energética y utilización de energía } \\
\text { renovable }\end{array}$ \\
\hline Recesión económica mundial & Plan de minimización y control de residuos \\
\hline Legislación & Reparación y atención a las emergencias ambientales \\
\hline Conflictos armados & Certificación del sistema de Gestión Medioambiental \\
\hline Estabilidad política & Responsabilidad social corporativa \\
\hline Nivel de industrialización del país & Defensa de los derechos humanos \\
\hline Nivel de corrupción & Ética profesional \\
\hline Clima del país & $\begin{array}{l}\text { Desarrollo profesional, atracción y retención de } \\
\text { talentos }\end{array}$ \\
\hline Recursos naturales & $\begin{array}{l}\text { Certificación del Sistema de prevención de riesgos } \\
\text { laborales }\end{array}$ \\
\hline Nivel de crecimiento económico & Buen gobierno corporativo \\
\hline Nivel educativo & $\begin{array}{l}\text { Transparencia e información en las prácticas de } \\
\text { gestión }\end{array}$ \\
\hline Desarrollo tecnológico del país & Política de control y gestión de riesgos \\
\hline Catástrofes naturales & Viabilidad económica financiera \\
\hline & Certificación del Sistema de gestión de calidad \\
\hline
\end{tabular}

Fuente: Fundación Entorno (2009)

Para la Fundación Entorno (2009), descubrir las causas y los efectos que pueden afectar la sostenibilidad es un reto para las empresas, aún más en el escenario global actual, cada vez más complejo, cambiante e incierto. Así, además de los aspectos que aparecen en la Tabla 2, se expresa como los factores mentales también influyen a la hora de la aplicación de la sostenibilidad. La empresa tradicional está pensada para obtener el máximo beneficio con el mínimo coste, y la sostenibilidad se considera un coste. A ello hay que sumarle las pocas iniciativas existentes hasta ahora, por lo tanto, la falta de ejemplos que sirvan de guía. Por lo general las empresas tienen miedo a lo desconocido y esto les impide avanzar.

\section{METODOLOGÍA}

La investigación que da origen a este artículo se ubica dentro de los estudios descriptivos, por cuanto se orienta a representar la información relacionada con los 


\section{Revista científica, arbitrada e indizada, bajo la modalidad electrónica.}

procedimientos para analizar las estrategias, la prospectiva y la sostenibilidad como enfoques gerenciales que permiten alcanzar la productividad y competitividad de las Pymes en un mercado global, donde se visualizan, caracterizan, conocen e interpretan aquellas situaciones o hechos reales presentes dentro de estas unidades de producción.

Es así como la investigación descriptiva "comprende la descripción, análisis, registro e interpretación de la naturaleza actual, y la composición o proceso de los fenómenos. El enfoque se hace sobre conclusiones dominantes o sobre como una persona, grupo o cosa se conduce o funciona en el presente" (Tamayo y Tamayo, 2009, p. 46). Por consiguiente, la investigación descriptiva "trabaja sobre realidades de hecho, y su característica fundamental es la de presentar una interpretación correcta. Igualmente, consiste en describir y analizar sistemáticamente características homogéneas o fenómenos estudiados sobre la realidad sea de individuos o comunidades" (Bavaresco, 2006, p. 26), citada por Pelekais et al., (2015).

De igual manera, el diseño fue de tipo no experimental, transversal descriptivo, dado que al analizar las estrategias, la prospectiva y la sostenibilidad como enfoques gerenciales que permiten alcanzar la productividad y competitividad de las Pymes en un mercado global, no se manipularon las variables, sino que se midieron en su contexto natural, por cuanto se recopilan los datos en un momento natural y se descarta el manejo intencional de las variables estrategia, prospectiva y sostenibilidad. Hernández et al., (2014), se refieren a los diseños de investigación no experimental en la siguiente cita:

Se realiza sin manipular deliberadamente las variables; además, definen a los diseños transversales descriptivos como aquellos que tienen como objetivo indagar la incidencia y los valores en que se manifiestan una o más variables (dentro del enfoque cuantitativo) o ubicar, categorizar y proporcionar una visión de una comunidad, un evento, un contexto, un fenómeno o una situación (describirla, como su nombre lo indica, (dentro del enfoque cualitativo). (p. 267).

En relación con la población, esta "es el conjunto total de unidades de análisis que se consideran en el estudio (nación, estados, grupos, comunidades, objetos, instituciones, asociaciones, actividades, acontecimientos, establecimientos, empresas, 


\section{Revista científica, arbitrada e indizada, bajo la modalidad electrónica.}

personas, individuos, entre otros), es decir, es la totalidad de los elementos que forman un conjunto." (Bavaresco, 2006, p. 91). También es definida como "el universo del estudio sobre el cual se pretende generalizar los resultados, está constituida por características o estratos similares pero concisos los cuales permiten distinguir dichos sujetos unos de otros". (Chávez, 2007, p. 162).

Para el presente estudio, la población está conformada por las micro, pequeñas y medianas empresas que hacen parte del sector de consumo masivo de la ciudad de Quibdó, Departamento del Chocó, Colombia, las cuales se encuentran registradas en la Cámara de Comercio, con un capital social promedio de $\$ 35.000 .000$. En tanto, las unidades informantes estuvieron representadas por las cadenas de supermercados Mercadiario y Confimax. El criterio de selección del grupo de informantes fue intencional, puesto que debió cumplir con los siguientes requisitos:

- Experiencia, habilidades, destrezas, reconocimiento, respeto y credibilidad dentro del sector de las Mipymes del sector de consumo masivo.

- Emprendedores de reconocida trayectoria y experiencia dentro del nivel empresarial de los referidos supermercados de estudio.

De esta manera, con relación a los escenarios donde se realizan los respectivos análisis establecidos dentro de este estudio, se destacan diferentes espacios estructurales, los cuales son de carácter logístico y operativo de los supermercados Mercadiario y Confimax, de la Ciudad de Quibdó, Chocó, en Colombia.

Para la recolección de la información, se diseñó una encuesta semiestructurada con base en el alcance del objetivo y los principales aspectos que relacionan los enfoques gerenciales de estrategia, la prospectiva y la sostenibilidad con el alcance de la productividad y competitividad de las Pymes. Estableciéndose métodos que permitieron prever que las preguntas no fueran repetidas o ambiguas.

La obtención de la información primaria se realizó inicialmente un contacto telefónico para sensibilizar y comprometer a los empresarios acerca de la importancia de la investigación. Luego se envió el formato de encuesta al correo electrónico de cada una de 


\section{Revista científica, arbitrada e indizada, bajo la modalidad electrónica.}

las gerencias de las empresas, con una carta de consentimiento informado y, de manera simultánea, se les proporcionó la opción de responder la encuesta mediante un formato digital. De esta manera, se adquirieron los datos necesarios $\mathrm{y}$, finalmente, fue posible obtener información de las dos gerencias de los supermercados Mercadiario y Confimax.

El análisis de la información se efectuó mediante estadística descriptiva. Esta también fue utilizada posteriormente con la finalidad de describir el comportamiento de las variables estrategia, prospectiva y sostenibilidad, mediante la distribución, tanto de frecuencias absolutas, como porcentuales, así como promedios estadísticos aritméticos correspondientes a cada uno de los indicadores utilizados en las respectivas dimensiones relacionadas al objetivo. Para ello se utilizó el programa Excel de Microsoft Windows, versión 2007.

Con respecto al análisis que determinaron los hallazgos, se estableció un baremo que contenía una escala de alternativas de respuestas utilizadas en el instrumento. Además, para la discusión e interpretación de los resultados del presente estudio, el investigador diseñó un cuadro de rango, intervalo como de categoría, para realizar la interpretación del análisis de los resultados de los indicadores y de las dimensiones a las cuales se les concede los valores en correspondencia a las alternativas siempre (4) casi siempre (3), a veces (2) y nunca (1). (Ver Tabla 3).

Tabla 3. Baremo para la categorización de los estadísticos de las variables

\begin{tabular}{|l|c|c|l|}
\hline \multicolumn{1}{|c|}{ Alternativas } & Intervalo & Categoría & \multicolumn{1}{|c|}{ Significancia } \\
\hline \hline Siempre (S) & $3,28 \geq \mathrm{X} \leq 4,00$ & Alto & $\begin{array}{l}\text { Alto desarrollo de las actividades en las } \\
\text { respectivas Variables estrategia, prospectiva } \\
\text { y sostenibilidad. }\end{array}$ \\
\hline \hline Casi Siempre (Cs) & $2,52 \geq \mathrm{X}<3,27$ & Moderado & $\begin{array}{l}\text { Desarrollo de las respectivas actividades } \\
\text { correspondiente a las Variables estrategia, } \\
\text { prospectiva y sostenibilidad, con deficiencias } \\
\text { en las frecuencias de ejecución de las mismas. }\end{array}$ \\
\hline \hline $\begin{array}{l}\text { Algunas veces } \\
\text { (Av) }\end{array}$ & $1,76 \geq \mathrm{X}<2,51$ & Bajo & $\begin{array}{l}\text { Presenta un alto número de debilidades y/u } \\
\text { omisiones en el desarrollo de las actividades } \\
\text { respecto a las Variables estrategia, } \\
\text { prospectiva y sostenibilidad. }\end{array}$ \\
\hline
\end{tabular}




\section{Revista científica, arbitrada e indizada, bajo la modalidad electrónica.}

\begin{tabular}{|c|c|c|l|}
\hline Alternativas & Intervalo & Categoría & \multicolumn{1}{|c|}{ Significancia } \\
\hline \hline Nunca $(\mathrm{N})$ & $1,00 \geq \mathrm{X}<1,75$ & Muy Bajo & $\begin{array}{l}\text { Poco o nada desarrollado de actividades } \\
\text { correspondiente a las Variables estrategia, } \\
\text { prospectiva y sostenibilidad. }\end{array}$ \\
\hline
\end{tabular}

Fuente: Elaboración propia. (2020).

\section{Hallazgos}

Dentro de este apartado se presentan los resultados producto de la metodología empleada, así como su relación con el objetivo, el cual consiste en analizar las estrategias, prospectiva y sostenibilidad como enfoques gerenciales que alcanzan la productividad y competitividad de las Mipymes en un mercado global. De esta manera, una empresa podrá alcanzar ventajas competitivas cuando cuenta con recursos y habilidades únicas que le permitan implementar estrategias de negocio superiores a las de sus rivales más cercanos. Así, estas ventajas competitivas pueden tener su origen en diversos aspectos, como la tecnología, el conocimiento, los costos, o la especialización en un segmento del mercado nacional e internacional.

Por consiguiente, cuando estas ventajas resisten al impacto o a las condiciones del entorno, las empresas logran mantenerse en el tiempo. De hecho, la visión basada en los recursos pone de relieve ciertas características que deben tener los recursos para ser fuentes de ventajas competitivas sostenibles, ya que estas provienen de capacidades dinámicas, las cuales son definidas como la habilidad de la empresa para integrar, construir y reconfigurar su fuente o apropiación de recursos, para de esta forma, responder rápidamente a los cambios del entorno. Por consiguiente, la capacidad para desarrollar nuevos recursos, además de procesos, se convierte en una de las más importantes fuentes de ventajas competitivas para las micro, pequeñas y medianas empresas.

En concordancia con planteamientos teóricos, la globalización y la liberalización económica han actuado como determinantes del fortalecimiento competitivo de las Mipymes, y en oportunidades han propiciado su internacionalización. En el caso particular de las empresas que comienzan a internacionalizarse, se produce una transferencia progresiva de sus recursos hacia las nuevas áreas geográficas a las que se expanden. 


\section{Revista científica, arbitrada e indizada, bajo la modalidad electrónica.}

Evidentemente, cada mercado tendrá sus propias características, por lo que los mismos recursos que pueden proporcionar una ventaja competitiva en un mercado, serán insuficientes para alcanzar competitividad en otro. Es precisamente aquí donde los enfoques gerenciales de estrategia, prospectiva y sostenibilidad generan su liderazgo o accionar en el alcance de la productividad y competitividad.

\section{CONCLUSIONES}

Finalizado el proceso de análisis de los resultados correspondiente a las estrategias, prospectiva y sostenibilidad como enfoques gerenciales que permiten alcanzar la productividad y competitividad de las micro, pequeñas y medianas empresas en un mercado global, se concluye que los empresarios y emprendedores reconocen que las estrategias les permiten establecerse y posicionarse en el mercado, en el cual la clave para permanecer, crecer y obtener el éxito o el fracaso está en implementar las estrategias adecuadas para generar ventajas, al igual diferenciación sobre sus competidores. Igualmente, su diseño y aplicación depende de las particularidades, recursos, capacidades y características propias de cada empresa.

De igual manera, se hace evidente la innovación como estrategia, la cual al interior de las empresas coadyuva en su crecimiento y genera un impacto significativo en el desarrollo de la economía y la sociedad, a partir de la creación de puestos de trabajo y de desarrollo humano. Las Mipymes obtienen un mayor margen de utilidad cuando adoptan e implementan a la innovación como una estrategia empresarial, generan operaciones más eficientes a la hora de generar ventajas competitivas y un mayor nivel de rendimiento.

Así mismo, la prospectiva influye y es abordada por las empresas como el proceso que determina la generación de estrategias relacionadas con la innovación, la estructura organizacional y el liderazgo. Esto les permite encontrar nuevas respuestas en los mercados en los cuales participan las empresas con sus productos o servicios, ya que a través de métodos prospectivos cualitativos o cuantitativos están tomando decisiones para el largo plazo. 


\section{Revista científica, arbitrada e indizada, bajo la modalidad electrónica.}

Es así que la prospectiva es vista por las Mipymes como una herramienta que facilita el conocimiento del contexto, la investigación de nuevos mercados, la actualización y el desarrollo de nuevos productos y servicios. Por consiguiente, la aplicación de métodos prospectivos está influyendo en trabajar con las capacidades humanas de exploración, descubrimiento y experimentación. De esta manera, con la prospectiva tomada por estas unidades de la producción como herramienta de gestión, se está corrigiendo y evitando riesgos o problemas que suelen presentarse dentro de un mercado global y competitivo.

Ahora bien, con respecto a la sostenibilidad, la globalización de los mercados ha generado que las economías se encuentren más dependientes unas de otras, y al mismo tiempo más susceptibles a las crisis, lo que genera en las micro, pequeñas y medianas empresas un mayor grado de incertidumbre. Así, estas se encuentran inmersas en un mercado globalizado, en el cual las inquietudes por el medio ambiente, las condiciones sociales y económicas del mercado están en condiciones de cambios, lo cual está influyendo en el aumento de necesidades en relación con sus grupos de interés. En este contexto, el desarrollo sostenible se está convirtiendo en un tipo de estrategia competitiva necesaria para la competitividad de las Mipymes dentro de un mercado global.

\section{REFERENCIAS BIBLIOGRÁFICAS}

Álvarez, W. J. (2014). ¿Es la innovación en las pymes colombianas una estrategia para el comercio internacional? Recuperado de: http://hdl.handle.net/10654/12272

Arango, A. (2005). Factores asociados con el éxito de las Pymes industriales en España. Universia Business Review, (8), 38 - 50.

Baena, P. G. (2004). Prospectiva Política Guía para su Comprensión y Práctica. México D.F.: Creative Commons.

Bavaresco, A. M. (2006). Proceso metodológico en la investigación: cómo hacer un diseño de investigación. Maracaibo: Editorial EDILUZ.

Beaver, G. (2007). The Strategy payoff for smaller Enterprise. Journal of Business strategy, $\mathrm{v}(6), 11-17$.

Burgelman, R. (2002). Strategy is Destiny: How Strategy Marketing shapes a Company future. New york: The Free press.

Chávez, N. (2007). Introducción a la Investigación Educativa. Maracaibo: Talleres Gráficos González.

Chiavenato, I. (2009). Comportamiento organizacional, la dinámica del éxito en las organizaciones. México D.F: Mc Graw Hill. 


\section{Revista científica, arbitrada e indizada, bajo la modalidad electrónica.}

Chirinos, Y. D. V., Meriño, V. H., Martínez, C. Y., \& Pérez, C. M. (2017). Emprendimiento sostenible para el desarrollo económico de las PYMES. Revista Espacios, 39(07), P.3- 4.

Culkin, N., \& Smith, D. (2000). An emotional business. A guide to understanding the motivations of small business decision takers. Canada, p. 145-150. Qualitative market research.

Dean, T., \& McMullen, J. (2007). Toward a theory of sustainable entrepreneurship: Reducing environmental degradation through entrepreneurial action. Journal of Business Venturing, 22(1), 50-76. doi: 10.1016/j.jbusvent.2005.09.003

Fundación Entorno. (2009). Entorno 2009: Informe sobre la gestión de la sostenibilidad en la empresa española. Madrid. Fundación Entorno.

Godet, M. (2011). La caja de herramientas de la prospectiva estratégica. Recuperado de http://es.laprospective.fr/dyn/espagnol/bo-lips-esp.pdf

Hernández, R., Fernández, C., Baptista, P. (2014). Metodología de la Investigación. México D.F.: Mc Graw-Hill.

Jones, G., \& George, J. (2006). Administración contemporánea. México D.F.: McGraw Hill.

Landázuri, S. N., \& Montenegro, N. J. (2018). The Strategic Approach of Michael Porter Applied to Mipymes: Reviata Hallazgos 21, Vol.3 The Ibarra - Ecuador Case. Pontificia Universidad Católica del Ecuador, Ibarra. P. 3- 4.

Medina, J., Becerra, S., Castaño, P. (2014). Prospectiva y política pública para el cambio estructural en América Latina y el Caribe. Editorial Naciones Unidas, Santiago de Chile.

Medina, J., Ortegón, E. (2006). Manual de prospectiva y decisión estratégica: Bases teóricas e instrumentos para América Latina y El Caribe. Santiago de Chile: Series manuales.

Miles, R., Snow, C., (2003). Organizational Strategy, Structure and Process. Nueva York: Stanford University Press.

Mojica, F. J. (2010). Introducción a la prospectiva estratégica para la competitividad empresarial. Trabajo de investigación, Universidad externado de Colombia. Bogotá. P. 5-10.

Naciones Unidas y la Comisión Económica para los Países de América Latina y el Caribe CEPAL. Proyecto de las MIPYMES en América Latina ''un frágil desempeño y nuevos desafíos para las políticas de fomento. 2018, Santiago de Chile.

Oslo M. (2012). The measurement of scientific and technological activities. Recuperado de https://www.oecd-ilibrary.org/docserver/9789264063525en.pdf?expires $=1587156877 \& \mathrm{id}=\mathrm{id} \&$ accname $=$ guest $\&$ checksum $=90086 \mathrm{BFF} 0 \mathrm{E} 866$ FFD6CFCB60A8A106222

Pelekais, C; El Kadi, O; Seijo, C; Neuman, N. (2015). El ABC de la investigación: Una aproximación teórico-práctica. Ediciones Astro Data. Venezuela. 


\section{Revista científica, arbitrada e indizada, bajo la modalidad electrónica.}

Pitre, R., Rodríguez, J., Hernández, H., Cardona, D. (2017). Emprendimiento competitivo y productivo como renovador del sector salud en la región Caribe. Revista Espacios, 38(42), 7-16.

Ponti, F. (2009). Innovación. 7 movimientos para construir una empresa innovadora. Barcelona: Granica.

Popper, R. (2008). Foresight Methodology: an overview and more Bonn. Manchester Editorial, Universidad de Manchester.

Sánchez, S. N. (2012). La sostenibilidad en el sector empresarial (tesis de maestría). Universitat Politécnica de Catalunya, Barcelona, España.

Tamayo y Tamayo, M. (2009). Metodología formal de la Investigación científica. LIMUSA, Noriega Editores. México.

Tarapuez, E., Guzmán, B. E., Hernández, R. P. (2016). Estrategia e innovación en las Mipymes colombianas ganadoras del premio Innova 2010-2013. Estudios Gerenciales, 32(139), 170-180.

Vroom, V. (2003). Educating managers for decision making and leadership. Management Decision, 41(10), 968-978

Wheelen, T., \& Hunger, D. (2013). Administración estratégica y política de negocios: Hacia la sostenibilidad global. Bogotá: Pearson. 